\title{
Hospital Length of Stay For COVID-19 Patients: Data-Driven Methods for Forward Planning
}

Bindu Vekaria ( $\square$ bindu.vekaria@manchester.ac.uk)

The University of Manchester https://orcid.org/0000-0001-6605-7956

\section{Christopher Overton}

The University of Manchester

\section{Arkadiusz Wisniowski}

The University of Manchester

\section{Shazaad Ahmad}

Manchester University NHS Foundation Trust

\section{Andrea Aparicio-Castro}

The University of Manchester

Jacob Curran-Sebastian

The University of Manchester

\section{Jane Eddleston}

Manchester University NHS Foundation Trust

\section{Neil Hanley}

The University of Manchester

\section{Thomas House}

The University of Manchester

Jihye Kim

The University of Manchester

\section{Wendy Olsen}

The University of Manchester

\section{Maria Pampaka}

The University of Manchester

\section{Lorenzo Pellis}

The University of Manchester

\section{Diego Perez Ruiz}

The University of Manchester

\section{John Schofield}

Manchester University NHS Foundation Trust

\section{Nick Shryane}

The University of Manchester

\section{Mark Elliot}




\section{Research article}

Keywords: COVID-19, Length of stay, Survival Analysis, England

Posted Date: October 1st, 2020

DOI: https://doi.org/10.21203/rs.3.rs-56855/v1

License: (c) (1) This work is licensed under a Creative Commons Attribution 4.0 International License. Read Full License

Version of Record: A version of this preprint was published at BMC Infectious Diseases on July 22nd, 2021. See the published version at https://doi.org/10.1186/s12879-021-06371-6. 


\title{
Hospital length of stay for COVID-19 patients: Data-driven methods for forward planning
}

\author{
Bindu Vekariaa ${ }^{2,3,6^{*}}$, Christopher Overton ${ }^{2,7^{\wedge}}$, Arkadiusz Wiśniowski ${ }^{1 \dagger}$, Shazaad Ahmad ${ }^{8}$, Andrea \\ Aparicio-Castro $^{1}$, Jacob Curran-Sebastian ${ }^{2,6}$, Jane Eddleston ${ }^{6}$, Neil A Hanley,6, Thomas House ${ }^{2,4,6}$, \\ Jihye Kim ${ }^{1}$, Wendy Olsen ${ }^{1}$, Maria Pampaka ${ }^{1}$, Lorenzo Pellis ${ }^{2}$, Diego Perez Ruiz ${ }^{1}$, John Schofield ${ }^{6}$, Nick \\ Shryane ${ }^{1}$ and Mark J Elliot ${ }^{1}$
}

\footnotetext{
${ }^{*}$ Correspondence:

bindu.vekaria@manchester.ac.uk

${ }^{2}$ Department of Mathematics,

University of Manchester, Oxford Road, M13 9PL Manchester, UK Full list of author information is available at the end of the article

$\dagger$ Co-corresponding author:

a.wisniowski@manchester.ac.uk

Co-corresponding author: christo-

pher.overton@manchester.ac.uk
}

\begin{abstract}
Background: Predicting hospital length of stay (LoS) for patients with COVID-19 infection is essential to ensure that adequate bed capacity can be provided without unnecessarily restricting care for patients with other conditions. Here, we demonstrate the utility of three complementary methods for predicting LoS using UK national- and hospital-level data.

Method: On a national scale, relevant patients were identified from the COVID-19 Hospitalisation in England Surveillance System (CHESS) reports. An Accelerated Failure Time (AFT) survival model and a truncation corrected method (TC), both with underlying Weibull distributions, were fitted to the data to estimate LoS from hospital admission date to an outcome (death or discharge) and from hospital admission date to Intensive Care Unit (ICU) admission date. In a second approach we fit a multi-state (MS) survival model to data directly from the Manchester University NHS Foundation Trust (MFT). We develop a planning tool that uses LoS estimates from these models to predict bed occupancy.
\end{abstract}

Results: All methods produced similar overall estimates of LoS for overall hospital stay, given a patient is not admitted to $\operatorname{ICU}(8.4,9.1$ and 9.3 days for AFT, TC and MS, respectively). Estimates differ more significantly between the local and national level when considering ICU. National estimates for ICU LoS from AFT and TC were 12.4 and 13.4 days, whereas in local data the MS method produced estimates of 22.8 days.

Conclusions: Given the complexity and partiality of different data sources and the rapidly evolving nature of the COVID-19 pandemic, it is most appropriate to use multiple analysis methods on multiple datasets. The AFT method accounts for censored cases, but does not allow for simultaneous consideration of different outcomes. The TC method does not include censored cases but does consider these different outcomes. The MS method can model complex pathways to different outcomes whilst accounting for censoring, but cannot handle non-random case missingness. Overall, we conclude that data-driven modelling approaches of LoS using these methods is useful in epidemic planning and management, and should be considered for widespread adoption throughout healthcare systems internationally where similar data resources exist.

Keywords: COVID-19; Length of stay; Survival Analysis; England 


\section{Background}

Since its emergence in December 2019 and classification in January 2020, SARS$\mathrm{CoV}-2$, the coronavirus that causes COVID-19, has spread rapidly, with 270 thousand confirmed infections in the UK by the end of May [1]. The exponential growth in the early days of each nation's outbreak has led to a doubling time of around three days [2]. Coupled with potentially high estimates of $R_{0}$ (the average number of new infections generated by an infected individual, in the absence of control measures and population acquired immunity) $[3,4,5]$, this has continued to have substantial impacts on healthcare systems across the world. Large growth rates and a delay between new infections and their detection, can lead to unexpected surges in bed demand. In order to restrict the spread of the pathogen, many countries have implemented mass quarantine (also known as lockdown) strategies, including England where the mass quarantine began on 23 March 2020 [6]. However, the effects of such interventions are not seen for at least a week [7], emphasising the need for careful, evidence-based, planning; particularly as the easing of mass quarantine measures is considered. In this context, the use of clinical care data to predict the demand for hospital and Intensive Care Unit (ICU) beds by patients presenting with COVID-19 is invaluable in optimising the effectiveness of planning by hospitals and therefore patient outcomes.

Understanding the impact of COVID-19 on hospital capacity breaks down into two core measurement tasks: first, to predict incidence (and thereby hospital admissions rates); and second, to estimate total length of stay (LoS) accurately allowing for variation in severity of disease and healthcare needs. The combination of these two measures can then be used to predict bed demand. This challenging task requires a careful modelling approach, particularly when high-quality data is limited within often fragmented healthcare systems. National datasets are crucial in understanding demand in hospitals across the country, but are flawed by amounts of record-level (or whole case) missingness that can bias the estimates. Routinely collected data generated by individual hospitals are, by definition, smaller and non-general but tend to be less prone to missingness and these can complement national data by providing insights for planning on a local level.

Estimating LoS has not been the primary focus of previous modelling; and studies that calculate LoS tend to use $a d-h o c$ approaches [8]. There is currently a lack of statistically principled modelling that accounts for both delays in patient outcomes and complex hospitalisation pathways. This problem is particularly important during the COVID-19 pandemic, since some groups of patients spend extended periods in hospital, and, for the most severe cases, in critical care. Furthermore, estimates of $\operatorname{LoS}$ that use deterministic models or observations drawn directly from data fail to take missingness into account $[9,10,11]$. Accurately calculating LoS therefore requires mathematical and statistical techniques that specifically address these issues.

In this paper, we present three methods for estimating LoS for patients with COVID-19 infection using both a nationally collected dataset and local data from a large inner city hospital Trust in the UK. The truncation corrected (TC) method corrects for the fact that observations are truncated at the day of reporting; accelerated failure time models (AFT) explicitly account for all observed LoS including 
those censored by not having seen the outcome; and the multi-state (MS) approach analyses LoS and takes into account dependence between outcomes such as discharge or death. Finally, we include measures of uncertainty in each of our model results, which should be incorporated into hospital planning strategies. With this principled approach, past data can be appropriately used to better prepare for the next phase of the COVID-19 pandemic.

\section{Methods}

Data

Outcome variables

We define two outcome events: death or discharge. All patients admitted to hospital will eventually experience one of these two outcomes. Then, we model LoS from hospital admission to either death or discharge. For the analysis shown in the Results section, we focus on LoS until any outcome, to facilitate comparison of the three methods. We account for whether the patient was in ICU or not and also estimate the LoS from hospital admission to ICU. In Appendix E, we further examine different outcomes using the TC and MS methods.

\section{CHESS}

The COVID-19 Hospitalisation in England Surveillance System (CHESS) collects reports from all NHS acute care hospital trusts to provide daily patient-level and aggregate data on COVID-19 hospitalisations. In the patient-level data, patients are followed through their hospitalisation pathway; the dates of various events are recorded, such as date of admission to hospital, date of admission to ICU and final outcome date.

\section{CHESS predictors}

We used four variables as predictors. First, sex, for which we removed patients with unknown values. Second, age, which we grouped into four categories $(<50,50-64$, $65-74,75+)$, and removed negative values and patients with a recorded age equal to zero (which did not seem genuine, based on the number of such cases and other factors such as comorbidities). Third, week of admission to hospital, which, in the TC model, we categorised in two groups: weeks 12 to 14 (i.e. from 16 March to 5 April 2020), and weeks 15 to 20 (from 6 April to 17 May 2020). In the AFT model, we used single week as a fixed effect predictor but present results for the two groups of admissions. Fourth, we used a binary indicator on whether a patient was admitted to ICU or not, and omitted the patients for whom this information was unknown. The resulting analytical sample is $n=6208$. Details of the data processing procedure are presented in Appendix C.

Routinely collected hospital data (MFT)

Routine data on the hospitalisation of patients were provided by Manchester University NHS Foundation Trust (MFT). MFT is the largest NHS Trust in England, comprising nine hospitals and accounting for approximately $2.5 \%$ of the National Health Service. For COVID-19 admission, there were three geographically distinct acute hospitals across South and Central Manchester: Manchester Royal Infirmary; 
Wythenshawe Hospital; and Trafford General Hospital. MFT serves the population of Greater Manchester, a large, ethnically diverse conurbation of approximately 2.8 million people. The data follow all patients through their clinical pathway for the duration of a single hospitalisation, and provide timings and lengths of stay in all critical care episodes. Patient data are complete unless patients are still in hospital, in which case they are censored.

\section{MFT data preparation}

Data were drawn from the Patient Administration System (PAS) and WardWatcher to join information on a patient's hospitalisation pathway and critical care episodes. Patients were selected from the MFT database if a swab was taken either on the day of their hospitalisation, or within 2 days of their hospital admission and tested positive for COVID-19. This was to discount any hospital-acquired cases since COVID19 positive cases who required hospitalisation due to non-COVID related health conditions may bias LoS estimates. We also excluded patients admitted for elective procedures requiring treatment for chronic illnesses such as dialysis. As a result of having multiple admissions close together, it was difficult to determine whether these cases were hospital-acquired or genuine COVID-19 admissions. The resulting sample included $n=834$ patients. The models based on the MFT data did not use information on predictors due to the smaller sample size, although from a methodological point of view these could be easily added to the models. Details of the data generating process are presented in Appendix A.

Data quality issues in length of stay data

There are several types of data quality issues that tend to be present in length of stay data and all are present in one or both of the two datasets. Some of these are a consequence of the reporting and data collection methods. Others are inherent to the nature of outbreaks, and will be present regardless of the data collection. Here, we present some key issues that need to be adjusted for, and discuss the implications of ignoring them. Accounting for these biases for COVID-19 can enable robust estimates that provide timely insight for policy and planning.

\section{Missing cases}

One issue with the CHESS dataset is missing cases. For example, the number of deaths recorded in CHESS is considerably less than the official figures. These also suffer from reporting lag issues but some indication about the level of missingness in CHESS can be obtained by comparing to the daily situation report (SITREP) data. On 26 May, there were 23504 deaths in hospital as attributable to COVID-19 in the SITREP data. This compares to an equivalent figure of 4071 in the raw CHESS data for the same day. This is indicative of case level missingness within CHESS of over $80 \%$. We discuss this issue in more detail in the discussion.

\section{Missing values on important variables}

Many rows in the data are incomplete. This is particularly problematic for data pertaining to outcome events: for example in some cases it is unclear whether a patient has not been discharged yet, or whether they have but the data have not 
been recorded. The amount of, and patterns of, missing patient information in the CHESS data is associated with the trust that reports the cases, with varying levels of missingness across different trusts (see Appendix B).

Censoring

In time-to-event studies, we observe a collection of individuals who are infected or have been exposed to infectious material. If these individuals could be followed indefinitely, the outcomes of all individuals would be observed. Therefore, this data can be used to determine the length of stay in the various compartments (states) of the disease progression pathway, as well as the probabilities of transitions into other states. However, during an outbreak we only observe individuals up until the most recent reporting date. This leads to right-censoring (e.g. [12]), when we only know the lower bound of duration until the next event in the pathway, and cannot accurately determine the length of time until their next transition nor to which state this will be. Thus, censoring may lead to the underestimation of the LoS.

\section{Truncation bias}

To remove the uncertainty around censored cases, we can instead condition our sample to only look at cases for whom the outcome has been observed. However, such a sample includes only cases with outcomes that occurred before the most recent reporting date, causing the sample to be truncated by the reporting date. This truncation leads to an over-expression of short LoS, since the recently infected individuals are only included if their LoS is short. Failing to account for this bias will underestimate the LoS of interest. [1]

Truncation is exacerbated by exponential growth in the early stages of an outbreak, since a higher proportion of cases will have been infected recently. By the final phase of an outbreak, truncation has a smaller effect since the majority of cases occurred sufficiently long ago to be unaffected by the truncation date. However, it will always be present as long as the epidemic is ongoing. Even in these late stages, whilst it may have a negligible impact across the whole outbreak, its effect might be of concern in certain scenarios, such as when using time as a predictor variable. In such a case, for events early in the epidemic, truncation will have very little effect, but for more recent events many cases may still be truncated. Such biases are often considered in the HIV literature [13, 14], due to the long infectious periods involved, but are often ignored for acute outbreaks. As alluded to in [15], this is potentially due to high quality data being available only after an explosive outbreak has finished, by which point these biases have little or no effect. However, when attempting to control ongoing epidemics, we require estimates of LoS distributions that are robust in the face of censoring and truncation.

\section{Survival analysis}

Survival analysis describes a collection of statistical procedures for which the outcome of interest is time until an event, often as a function of predictor variables $[16,17,18]$. A central assumption of most survival analytic methods is that the time

${ }^{[1]}$ This can be seen in Figure 2 by comparing the TC results to the LoS observed in the data. 
to event will have been censored for some observations, as discussed in Section Data quality issues.

Survival analysis may assume an underlying distribution for LoS in each state. Generally, LoS are observed to be right-skewed, so a distribution with this property should be used. In this paper, LoS is assumed to follow a Weibull distribution, which is a popular choice in survival analysis as it is robust in terms of violation of its assumptions. Therefore, the choice allows us to focus on the comparison between the different methods rather than the issues of model fit.

Figure 1 outlines the model used to represent the hospital pathways we consider in our analysis. Allowed transitions are indicated by directed arrows between any two states. Below, we outline the survival methods we selected for our analyses. Code for all methods is available at https://github.com/thomasallanhouse/ covid19-los.

\section{Accelerated Failure Time (AFT) model}

In the AFT model, rather than considering all of the hospitalisation pathways shown in Figure 1, we focus on predicting $\operatorname{LoS}$ in a given state, until another pre-specified event occurs. That is, we are interested in estimating the time between subsequent events in the pathway, such as from hospital admission to being admitted to ICU. We aggregate the final outcomes of death and discharge into a single outcome. This is necessary since it is not clear what the outcome will be for the censored cases in the CHESS data.

The response variable is the natural logarithm of the LoS, denoted by $\ln (t)$, which is explained by a vector of predictors $x$, with associated parameter vector $\beta$, and error term $\xi$ :

$$
\ln (t)=x \cdot \beta+\xi
$$

The assumed probability distribution of $\xi$ defines the hazard function, i.e. the probability that a case will experience an event at time $t$, given that they have not already experienced it until time $t[19,20]$. For $\xi$ we assumed a Weibull distribution, giving the hazard function $h(t)=p \lambda t^{p-1}$, where $\lambda=\exp (-p x \cdot \beta)$ and $p$ is the shape parameter defining the Weibull distribution. If $p>1$ the hazard is increasing over time, if $p<1$ the hazard is decreasing over time, and for $p=1$ the hazard is constant over time (which is equivalent to an exponential error term distribution). The predictors $x$ therefore increase or decrease the hazard and so accelerate (shorten) or decelerate (lengthen) the time to event, $t$.

The AFT model explicitly takes into account cases with right-censoring [20]. Thus, the model corrects for the potential underestimation of the LoS when only a portion of patients in the sample have observed the event.

A limitation of this simple model is when there is more than one potential event of interest [18]. In this study there were two events of interest: death and discharge. These are 'competing hazards', i.e. if a patient experienced one they were censored for experiencing the other. We could have run the model twice, once for each event, and treated patients who experienced the other event as being censored. This would have given unbiased results if the competing hazards were independent, but, for a 
given patient, as the hazard of death increases, it decreases for discharge, and vice versa. For this reason we considered a model of the joint event: death or discharge. ${ }^{[2]}$

We fitted separate models for patients who never entered ICU versus patients who did enter ICU at some point, as these groups were expected to have different baseline hazard functions. In all models, the predictors in $x$ were sex, age group and week of hospital admission (see Section CHESS).

All models were estimated using JAGS software implemented in the rjags R package [21]. For the shape parameter, we used a uniform prior, $p \sim \mathrm{U}(0,10)$, which represents our lack of information on this parameter. There is not a conjugate prior simultaneously for both the shape and scale parameters in the Weibull distribution [22]. An alternative specification for this prior is a Gamma distribution [23]. However, in our tests the results were virtually the same with both priors for $p$. The scale parameter $\lambda$ is specified via a prior for the predictors' coefficients $\beta$, which is multivariate normal with mean zero and variance equal to 10 , i.e. each element of $\beta$ is distributed as $\mathcal{N}(0,10) .{ }^{[3]}$

\section{Truncation corrected method}

In this method, we again focus on estimating the single LoS in a given state. We assume that LoS is given by a random variable $X$, drawn from a distribution with density function $f_{\theta}(\cdot)$, parameterised by a set of parameters $\theta$. In this analysis, we assume that $X$ is drawn from a Weibull distribution. We aim to determine the underlying parameters for this distribution by fitting the observed data using maximum likelihood estimation.

To use maximum likelihood estimation, we need to construct a likelihood function for the observed data. For each data point, the LoS is not directly observed. Instead, the arrival and departure dates and/or times that bracket the period of stay are observed. These correspond to two random variables, $E_{1}$ and $E_{2}$, linked by the $\mathrm{LoS}$ random variable, i.e. $E_{2}=E_{1}+X$. Instead of treating incomplete entries as censored, here we condition the data on observing both events. For example, if interested in the time from hospital admission to ICU admission, we condition on cases that have been admitted to hospital and to ICU. This introduces a truncation bias (See section Truncation bias), which needs to be corrected in the likelihood function. This approach does not take into account competing hazards, since we condition the data on observing the outcome of interest. However, this method enables LoS for different patient outcomes to be estimated, since censored cases are not included.

Our likelihood function is defined as the probability that the second event occurs on the observed date, given the time of the first event and that the second event must have occurred before the truncation date [14]. Therefore, we need to find

$$
f\left(E_{2}=e_{2} \mid\left\{E_{1}=e_{1}\right\} \cap\left\{E_{2} \leq T\right\}\right)=\frac{g_{E_{1}, E_{2}}\left(e_{1}, e_{2}\right)}{\int_{e_{1}}^{T} g_{E_{1}, E_{2}}\left(e_{1}, x\right) \mathrm{d} x},
$$

\footnotetext{
${ }^{[2]}$ This is not as counter-intuitive as it might sound since, although death is certainly not an equivalent outcome for the patient, our primary concern here is in length of stay regardless of outcome

[3] The model can also be estimated using maximum likelihood implemented in Stata 14 using the command streg (https://www.stata.com/manuals/ststreg.pdf)
} 
where $g_{E_{1}, E_{2}}$ is the joint distribution of $E_{1}$ and $E_{2}$. The time of the second event is the time of the first event plus the delay, $E_{2}=E_{1}+X$. Therefore $g_{E_{1}, E_{2}}=$ $g_{E_{2} \mid E_{1}}\left(e_{2} \mid e_{1}\right) g_{E_{1}}\left(e_{1}\right)=f_{\theta}\left(e_{2}-e_{1}\right) g_{E_{1}}\left(e_{1}\right)$, which gives

$$
f\left(E_{2}=e_{2} \mid\left\{E_{1}=e_{1}\right\} \cap\left\{E_{2} \leq T\right\}\right)=\frac{f_{\theta}\left(e_{2}-e_{1}\right) g_{E_{1}}\left(e_{1}\right)}{\int_{0}^{T-e_{1}} f_{\theta}(x) g_{E_{1}}\left(e_{1}\right) \mathrm{d} x}=\frac{f_{\theta}\left(e_{2}-e_{1}\right)}{\int_{0}^{T-e_{1}} f_{\theta}(x) \mathrm{d} x} .
$$

This can be maximised across all data points to find the maximum likelihood estimator for $\theta .{ }^{[4]}$

This method can be used to examine LoS to individual outcomes by specifying the events, e.g. specifying that the second event is a death. Additionally, the effect of predictor variables can be analysed by sub-setting the data and then modelling the LoS of each subset.

\section{Multi-state model}

Multi-state survival analysis extends the above two methods by permitting us to model the time to multiple outcome events in the presence of competing hazards $[24,25]$. Thus, we can model complex patient pathways upon admission to hospital.

Each permitted transition in Figure 1 is a survival model, where the instantaneous rate of transition from one state, $r$, to another state, $s$, otherwise known as the transition intensity, can be modelled similarly to hazard functions. For all transitions, we assume a Weibull AFT model, but this method can easily accommodate the use of any parametric or flexible parametric models used in standard survival analysis [19]. When there are $n_{r}$ competing events for state $r$, a patient entering state $r$ at time $t_{j}$ has their next event at $t_{j+1}$, which is given by the minimum of the survival times for the competing events, $s_{1}, \ldots, s_{n_{r}}$.

The data are formatted in such a way that we have a series of event times and LoS, each corresponding to a change in state. The last of these may be observed so that the patient has entered an absorbing state, i.e. they are discharged or dead, or right-censored if the patient is still in the hospital. Therefore, the data to inform the $n_{r}$ models consist of an indicator corresponding to whether or not the transition is observed or censored at $t_{j+1}$. In this format, we can separate the data by transition and fit a transition-specific Weibull model to each subset. ${ }^{[5]}$

We calculate time to each transition, and the confidence and prediction intervals for these, using forward simulation together with bootstrapping [26]. Individual survival times are simulated for patients using estimates from each fitted Weibull model, and iterating through all possible transitions until all patients have reached an absorbing state or are censored at a specified maximum follow-up time. More detail on the method, including equations, is provided in Appendix D.

\section{Results}

\section{Overall LoS}

Table 1 and Figure 2 show the overall estimated LoS for all three methods. The AFT and TC estimates were all based on models adjusted for the week of admission, sex,

${ }^{[4]}$ We maximise this using command fminsearch in MATLAB, but it is relatively simple to implement in any language. We provide both MATLAB and Python code in the Github repository.

${ }^{[5]}$ We estimate the parameter values by using maximum likelihood estimation in Python. 
and age group. The effect of sex was small and non-significant in all of the models, thus, we do not present breakdowns by it. Overall, the explanatory power of the predictor variables was only modest. They accounted for a maximum of 10 per cent of the variance in observed LoS in any of the AFT models. MS models were run without adjusting for any covariates.

Table 1 Overall length of stay estimates for England using the AFT and TC method, and for Manchester trusts using the MS method. Source: own elaboration using CHESS and MFT data.

\begin{tabular}{ll|ccc}
\hline Method & Hospital trajectory & Mean & SD & N \\
\hline TC & Hospital admission to outcome (no ICU) & 9.1 & 9.5 & 2794 \\
TC & Hospital admission to outcome (via ICU) & 17.3 & 13.1 & 2517 \\
TC & ICU entry to ICU exit & 13.4 & 13.8 & 1809 \\
TC & Hospital admission to ICU entry & 2.0 & 2.7 & 2983 \\
\hline AFT & Hospital admission to outcome (no ICU) & 8.4 & 8.9 & 2805 \\
AFT & Hospital admission to outcome (via ICU) & 16.2 & 12.0 & 2555 \\
AFT & ICU entry to ICU exit & 12.4 & 12.8 & 1809 \\
AFT & Hospital admission to ICU entry & 2.0 & 2.7 & 2983 \\
\hline Multistate & Hospital admission to outcome (no ICU) & 9.3 & 10.0 & 839 \\
Multistate & Hospital admission to outcome (via ICU) & 35.2 & 25.0 & 48 \\
Multistate & ICU entry to ICU exit & 22.8 & 18.6 & 57 \\
Multistate & Hospital admission to ICU entry & 4.0 & 6.5 & 42 \\
\hline
\end{tabular}

CHESS data for England

For the ICU patients (Hospital to Outcome via ICU), the shape parameters in AFT and TC methods were larger than one, implying the baseline hazard increased over time. For the non-ICU patients and LoS within the ICU, the baseline hazard remains constant in the AFT model and is slowly decreasing in TC, whereas for the Hospital to ICU admission it is decreasing in both models.

Overall, for hospital admission to final outcome, the mean $\operatorname{LoS}$ for patients not admitted to ICU was shorter, with an AFT mean of 8.4 (TC mean: 9.1) days, than that of patients who were admitted to ICU at some point, with an AFT mean of 16.2 (TC mean: 17.3) days. ICU admission was estimated to take 2.0 (2.0) days from hospital admission, and ICU patients were estimated to spend an average of 12.4 (13.4) days in ICU.

Standard Deviations (SD) of the estimated LoS are presented in Table 1 whereas Predictive Intervals (PIs) for the LoS in AFT and TC methods are shown in Figure 2. The standard deviations (SD) for both the AFT and TC models are remarkably similar in depicting the large variability in the observed LoS. With the exception of the LoS from the hospital admission to outcome via ICU, all SD suggest that the waiting times till outcome are approximately exponentially distributed.

MFT data

Similarly to AFT and TC methods, in the MS approach, we used a Weibull distribution for each of the transition times between states in Figure 1. Then, using fitted parameters, we used 400 bootstraps and $>10^{5}$ forward simulations in order to obtain estimates of the mean lengths of stay in each state, given each transition. 
The MFT data based results (comparable with trajectories obtained using CHESS dataset with AFT and TC models) are presented in Figure 2 Table 1, along the summaries of the data.

As with the AFT and TC methods, LoS for patients admitted to ICU is longer, with a mean of 35.2 days, than that of patients not admitted to ICU, with a mean of 9.3 days. ICU admission was estimated to take 4.0 days from hospital admission and ICU patients were estimated to spend an average of 22.8 days in critical care. Taking into consideration competing hazards between stepdown and death, our mean LoS estimate for a patient in ICU is between 17.4 and 24.8 days (Table A1 in Appendix E), though in the data we observe people that have much longer critical care periods ( $20 \%$ of patients have over 40 days in an ICU).

In our simulation, we allow patients to enter an ICU immediately upon their admission to hospital, (as such cases can be observed in the data). Our model admits anyone who is sent to an ICU within half a day of being hospitalised directly into the ICU. This means that, in reality, there is a subset of patients $(n=21)$ with LoS in the ward that is less than 0.5 days before ICU admission. We find that admitting such patients directly into ICU when simulating patient pathways does not significantly bias our estimates, but permits a more successful replication of the observed number of patients in each state over the course of the outbreak.

\section{Planning with LoS}

Figure 3 predicts bed occupancy in acute ward and ICU after running our simulator with the parameter estimates of all three methods. The red and blue lines represent the implementation of, and relaxation of mass quarantine, respectively. We simulate hospital admissions from 23 February, first assuming exponential growth with a doubling time of 3 days, followed by exponential decay shortly after the implementation of mass quarantine. Following the blue line, we plan for a reasonable worst case scenario, and so assume a slower growth in cases with a doubling time of 15 days. Changing the assumptions used to generate hospital admissions allows us to predict and plan for any scenario of interest.

The estimates from the AFT and TC methods yield similar predictions of bed occupancy and total observed outcomes. The MS model also gives similar predictions for acute ward and outcome but differs for ICU. The peak in bed occupancy in ICU in the MS output occurs roughly two weeks later than in the AFT and TC model outputs, and there is a slower decline after the peak. This is caused by the larger LoS estimates for the MS models as seen in Table 1 and Figure 2.

\section{The effect of predictors - England}

In Figure 4 and Table 2, we present the estimates of LoS broken down by two main predictors: age and week of admission. The mean waiting time from hospital admission to ICU entry (first column of Figure 4) is around two days irrespective of age. For hospital admission to outcome without ICU stay (second column of Figure 4), increasing age raises the length of stay, with length of stay around five days for the youngest age group and twelve days for the oldest, irrespective of the AFT or TC model. For individuals who go via ICU (third column of Figure 4), the 
pattern with age is less clear ${ }^{[6]}$. For the first three age groups, the length of stay is roughly similar (especially AFT model), with a slight decrease in the oldest age group with respect to the first two. The $75+$ age group, however, has a much shorter length of stay. A similar pattern is observed for mean LoS from ICU admission to ICU exit (fourth column of Figure 4).

Considering the week of admission as a predictor variable, there is less variability in LoS than in LoS disaggregated by age. For a great majority of hospital trajectories, the mean LoS seems to have decreased by, on average, 16 per cent, depending on the age group and the method used. This could be explained by potential behavioural changes in the later admission weeks. Firstly, after mass quarantine progressed individuals may have waited longer before presenting at hospital. Secondly, treatment policy has changed over the course of the outbreak, with the criteria for discharge being relaxed to ensure hospitals had capacity. Nonetheless, we also note large variability in predicted LoS both in earlier and later weeks under study.

\section{Discussion}

\section{Analysis of results}

Comparison of the three different models

In this study, we have presented three methods for estimating the LoS of patients with COVID19 infection. Overall, The AFT and TC methods produced similar estimates for LoS for all four hospital trajectories. This is reassuring and forms an effective cross-validation of both methods and results.

The estimated mean LoS from the AFT model are shorter by around one day than the TC means, except for the Hospital-ICU entry. This might be due to the exclusion of potentially censored cases in the AFT method ${ }^{[7]}$, since it was not clear these were genuinely censored or incomplete data entries. Both methods also yielded similar predictive uncertainty about the LoS, with TC producing slightly wider predictive intervals than the AFT method. This might be explained by the explicit inclusion of the predictors in the AFT model with a joint assessment of their effect on the LoS. The TC method assumes independence between predictors and is applied to the subsets of CHESS data disaggregated by the predictor categories.

There were large differences in predicted ICU LoS between the two CHESS based methods and the MS method, notably LoS in ICU. The mean estimates derived using AFT and TC methods (12-13 days) were 5-6 days less than those from the MS method. The predictive intervals overlap suggesting the variability in LoS is large. However, given the focus of the paper is on comparison, and bearing in mind the MFT data is an effective census of the MFT patients and therefore that estimates is reliable in terms of the mapping of the data to the population, it is valuable to consider possible explanations for the difference in the point estimates.

These differences may reflect several substantive factors. First, the MFT is one of five adult centres in the UK to have an extracorporeal membrane oxygenation

${ }^{[6]}$ This is likely to be caused by the LoS to death (via ICU) following the opposite pattern to the LoS to discharge (via ICU). Younger age groups appear to have a longer duration until death on ICU and a shorter duration before discharge. These patterns seemingly cancel out when looking at the LoS until any outcome. This analysis is not shown here since we are focusing on length of stay in hospital rather than different outcomes.

${ }^{[7]}$ For the ICU entry to ICU exit trajectory there were $n=108$ censored cases included in the model. For the trajectory from hospital admission to outcome via ICU: $n=43$, and without ICU: $n=14$. There were no censored cases in hospital admission to ICU entry. 
Table 2 Length of stay estimates with predictor variables for AFT and TC methods. Sample sizes differ due to the inclusion of censored observations in the AFT method. Source: own elaboration using CHESS data for England.

\begin{tabular}{|c|c|c|c|c|c|c|c|c|}
\hline \multirow[b]{2}{*}{ Trajectory } & \multirow[b]{2}{*}{ Age } & \multirow[b]{2}{*}{ Weeks } & \multicolumn{3}{|c|}{ AFT model } & \multicolumn{3}{|c|}{ TC model } \\
\hline & & & Mean & SD & $\mathrm{N}$ & Mean & SD & $\mathrm{N}$ \\
\hline \multirow{8}{*}{$\begin{array}{l}\text { Hospital } \\
\text { admission to } \\
\text { outcome } \\
\text { (no ICU) }\end{array}$} & \multirow[t]{2}{*}{1 to 49} & 12 to 14 & 4.9 & 4.8 & 146 & 5.1 & 6.5 & 146 \\
\hline & & 15 to 20 & 3.7 & 3.6 & 210 & 3.6 & 4.1 & 210 \\
\hline & \multirow[t]{2}{*}{50 to 64} & 12 to 14 & 7.3 & 7.2 & 223 & 7.0 & 7.4 & 223 \\
\hline & & 15 to 20 & 5.6 & 5.4 & 304 & 5.9 & 5.9 & 304 \\
\hline & \multirow[t]{2}{*}{65 to 74} & 12 to 14 & 10.6 & 10.4 & 204 & 11.0 & 10.5 & 204 \\
\hline & & 15 to 20 & 8.1 & 7.9 & 270 & 8.3 & 7.7 & 266 \\
\hline & \multirow[t]{2}{*}{$75+$} & 12 to 14 & 11.7 & 11.4 & 609 & 11.7 & 10.9 & 607 \\
\hline & & 15 to 20 & 8.8 & 8.6 & 839 & 10.0 & 9.4 & 834 \\
\hline \multirow{8}{*}{$\begin{array}{l}\text { Hospital } \\
\text { admission to } \\
\text { outcome } \\
\text { (via ICU) }\end{array}$} & \multirow[t]{2}{*}{1 to 49} & 12 to 14 & 17.5 & 12.5 & 312 & 17.5 & 11.8 & 312 \\
\hline & & 15 to 20 & 14.3 & 10.5 & 267 & 17.8 & 14.0 & 262 \\
\hline & \multirow[t]{2}{*}{50 to 64} & 12 to 14 & 18.8 & 13.4 & 641 & 19.5 & 14.3 & 626 \\
\hline & & 15 to 20 & 15.7 & 11.5 & 467 & 17.0 & 12.1 & 455 \\
\hline & \multirow[t]{2}{*}{65 to 74} & 12 to 14 & 16.8 & 12.0 & 391 & 17.1 & 13.5 & 388 \\
\hline & & 15 to 20 & 13.9 & 10.2 & 225 & 14.9 & 10.2 & 223 \\
\hline & \multirow[t]{2}{*}{$75+$} & 12 to 14 & 13.3 & 9.5 & 161 & 12.6 & 10.8 & 161 \\
\hline & & 15 to 20 & 10.2 & 7.6 & 91 & 11.3 & 8.9 & 90 \\
\hline \multirow{8}{*}{$\begin{array}{l}\text { ICU entry to } \\
\text { ICU exit }\end{array}$} & \multirow[t]{2}{*}{1 to 49} & 12 to 14 & 13.0 & 12.8 & 239 & 13.2 & 14.0 & 239 \\
\hline & & 15 to 20 & 10.0 & 10.0 & 210 & 12.7 & 14.5 & 210 \\
\hline & \multirow[t]{2}{*}{50 to 64} & 12 to 14 & 15.4 & 15.2 & 468 & 15.4 & 14.2 & 468 \\
\hline & & 15 to 20 & 12.0 & 12.0 & 337 & 13.6 & 13.8 & 337 \\
\hline & \multirow[t]{2}{*}{65 to 74} & 12 to 14 & 13.6 & 13.4 & 237 & 13.6 & 12.5 & 237 \\
\hline & & 15 to 20 & 10.4 & 10.4 & 152 & 11.4 & 11.4 & 152 \\
\hline & \multirow[t]{2}{*}{$75+$} & 12 to 14 & 7.6 & 7.5 & 109 & 8.1 & 8.9 & 109 \\
\hline & & 15 to 20 & 5.5 & 5.6 & 57 & 5.0 & 5.9 & 57 \\
\hline \multirow{8}{*}{$\begin{array}{l}\text { Hospital } \\
\text { admission to } \\
\text { ICU entry }\end{array}$} & \multirow[t]{2}{*}{1 to 49} & 12 to 14 & 2.0 & 2.6 & 340 & 1.9 & 2.5 & 340 \\
\hline & & 15 to 20 & 1.7 & 2.3 & 336 & 1.8 & 2.4 & 336 \\
\hline & \multirow[t]{2}{*}{50 to 64} & 12 to 14 & 2.2 & 2.9 & 732 & 2.4 & 3.3 & 732 \\
\hline & & 15 to 20 & 2.0 & 2.7 & 610 & 1.8 & 2.2 & 610 \\
\hline & \multirow[t]{2}{*}{65 to 74} & 12 to 14 & 2.2 & 2.9 & 421 & 2.1 & 2.9 & 421 \\
\hline & & 15 to 20 & 1.9 & 2.6 & 276 & 1.9 & 2.4 & 276 \\
\hline & \multirow[t]{2}{*}{$75+$} & 12 to 14 & 2.4 & 3.1 & 168 & 1.9 & 2.4 & 168 \\
\hline & & 15 to 20 & 2.1 & 2.8 & 100 & 2.6 & 3.2 & 100 \\
\hline
\end{tabular}

(ECMO) unit. This is a unit admitting severe patients that require more than mechanical ventilation to keep them alive. It takes over a patient's heart and lung functions by oxygenating blood outside of the body, and results in very long LoS. As MFT receives patients needing ECMO from across the Northwest, they account for roughly $20 \%$ of the total patients requiring critical care in the MFT dataset. This is much higher than the proportion of ECMO patients to standard ICU patients 
in the CHESS dataset (which is around 2.6\%). ${ }^{[8]}$. We examined this explanation further by running all models without the patients using ECMO for ICU entry-ICU exit. For the multi-state model using the MFT data the predicted mean LoS is 21.9 $(\mathrm{SD}=16.9)$. For the AFT model on the CHESS sample without ECMO patients $(\mathrm{N}=1762)$ the results are virtually the same as with ECMO. The predicted mean LoS is $12.3(\mathrm{SD}=12.8)$. For the TC method the predicted mean LOS is $13.2(\mathrm{SD}=$ 13.7)

Second, the underlying data were different: the AFT and TC models used the country-wide but very incomplete CHESS data, whereas the multi-state model was based on data from just one NHS trust, but largely free of missing data.

Third, differences in excess bed demand from trust to trust potentially further explain discrepancies in our estimates. For trusts experiencing significant increases in demand, it is possible that they do not have the ability or resources to accurately generate daily CHESS reports which are collected in addition to routinely collected data (see Appendix A). This partially explains the case-missingness in the CHESS data. On 26 May 2020 the CHESS data after cleaning showed 4,676 (32\%) had reached ICU, compared with $6 \%$ in MFT data. Given the already small sample size of the MFT data, the ICU sub-sample is relatively small and sensitive to noise.

In order to sensitivity check the findings for the differences in the data, we evaluated the AFT model and TC method using CHESS data for the Manchester Trust only. MFT contributed 53 cases with recorded durations in ICU to CHESS. Running the AFT model on these cases gave a predicted ICU LoS of 16.5 days $(\mathrm{SD}=17.3)$. For the TC method, the predicted mean was $16.1(\mathrm{SD}=16.7)$. The estimated LoS were longer than the full-sample CHESS estimates but still shorter than the predicted LoS from the MS models.

A complicating factor here is that during early 2020 the North Manchester General Hospital which is part of the Pennine Acute Hospitals NHS Trust was transferred to MFT under a management agreement. This change appears to be represented in CHESS but not in the MFT data themselves. Unfortunately, we are not able to distinguish those cases within CHESS.

All methods captured the variability in the data and reflected it in the predictive distributions. This uncertainty should be taken into account when planning for the number of beds during the pandemic. For example, upper bounds of the predictive intervals can be used to construct extreme-case scenarios for the beds occupancy. These can be fed into the multi-state model to predict the number of patients in hospital at various stages of the pandemic (Figure 3).

In the main LoS analysis above, we did not distinguish between different outcomes, such as death or discharge. Particularly in ICU, the baseline hazards for these competing hazards may be strongly diverging over time. In Appendix F, we analyse the length of stay for given outcomes using the TC and MS methods, finding that in general the length of stay to discharge is longer than to death.

\section{Limitations of research}

The CHESS dataset suffers from large amounts of case-missingness, which may lead to bias in the estimates. There appear to be three types of this. Update delay

${ }^{[8]}$ Also, since many of the ECMO patients are censored due to requiring long periods of care, this could explain why the MS model slightly overestimates LoS in ICU in Figure 6 
where a record has not been updated (with a transition) which may lead to incorrect censoring. This leads to the patient being removed from the data for some of the models. Reporting delay where a patient does not appear in the data at all until sometime after their admission. Non-reporting where no report is ever made on a patient. All three of these may cause bias in the models if they are correlated with either LoS or with extraneous variables (that are not controlled for within a given model). Another limitation of both datasets was that only cases of COVID-19 infection that led to hospital admission were included in the data. During March 2020, the hospitalised patients in England were considered to reflect the underlying population of patients with severe COVID-19 infection, but by 14 April, care-home deaths reported on death certificates caused a revision of views [27]. Those severe cases not attending hospital and COVID-19-related deaths outside of hospital may have different properties from hospitalised patients and deaths. So care should be taken in extrapolating the findings to general statements about disease progression outside of the hospital setting. Given that the goal here was to model length of stay in hospital this is less of a concern. However, change in hospitilastion practice could lead to changes in the estimates that the models produce.

Our models were also limited by the missing values in the CHESS data. A notable limitation was that around half of the cases did not have their final outcome or current status recorded. We did not know if this implied that the patient was still in hospital or whether it was an omission or whether this was a result of update delay. In either case, we had no reliable way to estimate the last time point at which the patient was observed to be in hospital, and thus these patients could not contribute to the LoS estimates. If those patients with missing data for key dates were mostly still in hospital, then the CHESS-based LoS estimated by using the AFT model are likely to represent underestimates of the true but unrecorded LoS for all patients.

Compared with the AFT model, the TC method should, in theory, be less sensitive to this issue since it ignores censored cases. However, this method relies on sufficient tail observations being recorded. With the long duration of this study, sufficient tail observations should be included, though if the majority of missing data are omitted rather than censored, this may lead to bias in the method. For example, omitted cases might correspond disproportionately to tail observations, which would cause the truncation corrected method to underestimate length of stay. We cannot determine this from the missing data, which may cause inaccuracies in the estimates.

The strength of bias due to the truncation and censoring varies depending on the phase of the epidemic, with it having a large impact during exponential growth and lessening impact during the decay phase. The data used in this analysis is from the decay phase, so the truncation bias does not have a huge impact, and ignoring this bias would underestimate LoS by up to two days (using TC method). However, for a sample earlier in the outbreak, this underestimation may be amplified, as well as the difference in model performance. This is also true for censoring biases, since early in the outbreak the majority of cases will have censored outcomes. A large number of right-censored cases would lead to relatively large values of LoS when using the AFT model. For the purposes of this paper, we have opted not to investigate the performance of each model at different sampling dates. This is to focus on 
the presentation of the different methods using a single illustrative example to improve clarity. Future research could extend this in several ways including running iteratively through the data available on different dates modelling the impact of truncation, censoring, reporting and updating lags as the epidemic progresses.

Another issue is that clustering of patients within the NHS trusts, which were at different stages of the epidemic at different times leading to variations in pressure on capacity, could mean that there are spatial-temporal interactions in the processes driving LoS which are not captured in the models. Further, these may in turn interact with the data generating processes for CHESS with more non-reporting and reporting delays likely during high demand times. These issues could have unpredictable effects on the estimates of LoS.

With respect to the MFT data, most limitations arise due to the small absolute sample size. The multi-state method requires seeing an adequate number of patients for each state transition before any reliable modelling can take place. Indeed, although it is clinically known that stepdown to mortality is a valid transition, after applying our exclusion criteria, there were no observations of this transition occurring for patients with COVID-19 infection within this Manchester Trust. The analysis conducted in this paper therefore excluded this transition, and it is not possible to see how this influences overall hospital LoS of those patients who have an ICU episode during their hospitalisation. Together with uncommonly long ICU periods, the relative delay in the Manchester epidemic compared to other parts of the country means that MFT patients with long critical care spells are either still in ICU or only just starting to move onto stepdown. Given more weeks of data, we might be able to include stepdown to mortality in our model.

The above suggests differences between the estimates of LoS for the two datasets may therefore be due more to differences in the available data than differences in the statistical methods per se. It is important to acknowledge these uncertainties in the data when interpreting length of stay estimates. We further note that not only would we obtain more power in predictions through a larger amount of complete data, but also a better understanding of how the complex interactions between the virus and background risk factors affect disease severity.

\section{Conclusions}

In this paper and its supporting materials, we provide a freely accessible set of models and tools to estimate LoS with an application to patients with COVID19 infection. Together with a prediction of hospital admissions, LoS predictions can be implemented to provide organisational support within hospitals to ensure the demand for hospital and, in particular, ventilated ICU beds does not exceed availability. The models use routinely collected hospital data which are available within many national healthcare systems. Thus we anticipate our approaches will have utility across diverse healthcare systems in many different countries. 


\section{Abbreviations}

AFT: Accelerated Failure Time; CHESS: COVID-19 Hospitalisation in England Surveillance System; ECMO:

Extracorporeal Membrane Oxygenation; ICU: Intensive Care Unit; LoS: Length of Stay; MFT: Manchester University NHS Foundation Trust; MS: Multi-State; PAS: Patient Administration System; PI: Predictive Intervals; SD:

Standard Deviation; TC: Truncation Corrected

Declarations

Ethics approval and consent to participate

The University of Manchester Ethics Decision Tool ruled that no formal ethics approval was required for this particular study, and it would be unethical to use the time of an ethics committee to confirm this, given that the project involved secondary use of data subject that were functionally anonymised, and that the data were being used for the purposes that were compatible with the original consent of the data subjects with full permission of the data owner.

Consent for publication

Not applicable.

Availability of data and material

The data involved in this work is sensitive and therefore not publicly available. Code and parameter estimates are publicly available at https://github.com/thomasallanhouse/covid19-los.

Competing interests

The authors declare that they have no competing interests.

Funding

TH receives support from the Royal Society (Grant Number INF/R2/180067) and the Alan Turing Institute for Data Science and Artificial Intelligence. LP and CO are funded by the Wellcome Trust and the Royal Society (Grant Number 202562/Z/16/Z). BV is funded by an MRC Doctoral Partnership grant (Grant Number MR/R502236/1)

Authors' contributions

$\mathrm{BV}, \mathrm{CO}, \mathrm{AW}, \mathrm{TH}$ and ME had roles in the study design, data analysis, data interpretation, literature search, and writing of the manuscript. AAC, JCS, JK, WO, DPR, MP, LP, and NS had roles in data analysis, data interpretation and study design. SA and JE provided clinical expertise and information. JS, JE, NH assisted with data collection and study design. All authors reviewed and commented on at least one draft of the paper and approved the final manuscript.

Acknowledgements

Not applicable.

\section{Author details}

${ }^{1}$ Department of Social Statistics, School of Social Sciences, University of Manchester, Oxford Road, M13 9PL Manchester, UK. ${ }^{2}$ Department of Mathematics, University of Manchester, Oxford Road, M13 9PL Manchester, UK.

${ }^{3}$ Division of Informatics, Imaging and Data Science, Faculty of Biology, Medicine and Health, University of Manchester, Manchester Academic Health Science Centre, Oxford Road, M13 9PL Manchester, UK. ${ }^{4}$ IBM Research, Hartree Centre, Daresbury, UK. ${ }^{5}$ Division of Diabetes, Endocrinology \& Gastroenterology, School of Medical Sciences, Faculty of Biology, Medicine \& Health, University of Manchester, Oxford Road, M13 9PT Manchester, UK. ${ }^{6}$ Clinical Data Science Unit, Manchester University NHS Foundation Trust, Oxford Road, M13 9WU Manchester, UK. ${ }^{7}$ Department of Mathematics, University of Liverpool, Peach Street, L69 7ZL Liverpool, UK. ${ }^{8}$ Department of Virology, Manchester Medical Microbiology Partnership, Manchester Foundation Trust, Manchester Academic Health Sciences Centre, Oxford Road, M13 9WU Manchester, UK.

\section{References}

1. UK Government. Slides and datasets to accompany coronavirus press conferences [Webpage]; 2020. Available from: https://www.gov.uk/government/publications/ slides-and-datasets-to-accompany-coronavirus-press-conference-29-may-2020. Accessed 04/06/2020.

2. Pellis L, Scarabel F, Stage HB, Overton CE, Chappell LH, Lythgoe KA, et al. Challenges in control of Covid-19: short doubling time and long delay to effect of interventions. arXiv preprint arXiv:200400117. 2020;

3. Liu Y, Gayle AA, Wilder-Smith A, Rocklöv J. The reproductive number of COVID-19 is higher compared to SARS coronavirus. Journal of travel medicine. 2020;

4. Mahase E. China coronavirus: what do we know so far? BMJ. 2020;368. Available from: https://www.bmj.com/content/368/bmj.m308.

5. Sridhar D, Majumder MS. Modelling the pandemic. BMJ. 2020;369. Available from: https://www. bmj.com/content/369/bmj.m1567.

6. The Health Foundation. COVID-19 Policy Tracker [Webpage]; 2020. Available from: https://www.health.org.uk/news-and-comment/charts-and-infographics/covid-19-policy-tracker. Accessed 04/06/2020.

7. Pellis L, Scarabel F, Stage HB, Overton CE, Chappell LHK, Lythgoe KA, et al. Challenges in control of Covid-19: short doubling time and long delay to effect of interventions. medRxiv. 2020;. 
8. Rees EM, Nightingale ES, Jafari Y, Waterlow NR, Clifford S, Pearson CA, et al. COVID-19 length of hospital stay: a systematic review and data synthesis. medRxiv. 2020;.

9. Ferstad JO, Gu AJ, Lee RY, Thapa I, Shin AY, Salomon JA, et al. A model to forecast regional demand for COVID-19 related hospital beds. medRxiv. 2020;

10. Weissman GE, Crane-Droesch A, Chivers C, Luong T, Hanish A, Levy MZ, et al. Locally informed simulation to predict hospital capacity needs during the COVID-19 pandemic. Annals of internal medicine. 2020;

11. Massonnaud C, Roux J, Crépey P. COVID-19: Forecasting short term hospital needs in France. medRxiv. 2020;

12. Sá C, Dismuke CE, Guimarães P. Survival analysis and competing risk models of hospital length of stay and discharge destination: the effect of distributional assumptions. Health Services and Outcomes Research Methodology. 2007;7(3-4):109-124.

13. Kalbfleisch J, Lawless J. Regression models for right truncated data with applications to AIDS incubation times and reporting lags. Statistica Sinica. 1991;p. 19-32.

14. Sun J. Empirical estimation of a distribution function with truncated and doubly interval-censored data and its application to AIDS studies. Biometrics. 1995;p. 1096-1104.

15. Becker NG, Britton T. Statistical studies of infectious disease incidence. Journal of the Royal Statistical Society: Series B (Statistical Methodology). 1999;61(2):287-307.

16. Cox DR. Regression models and life-tables. Journal of the Royal Statistical Society: Series B (Methodological). 1972;34(2):187-202.

17. Wei LJ. The accelerated failure time model: a useful alternative to the Cox regression model in survival analysis. Statistics in medicine. 1992;11(14-15):1871-1879.

18. Kalbfleisch JD, Prentice RL. The statistical analysis of failure time data. vol. 360. John Wiley \& Sons; 2011

19. Crowther MJ, Lambert PC. Parametric multistate survival models: flexible modelling allowing transition-specific distributions with application to estimating clinically useful measures of effect differences. Statistics in medicine. 2017;36(29):4719-4742.

20. Carroll KJ. On the use and utility of the Weibull model in the analysis of survival data. Controlled clinical trials. 2003;24(6):682-701.

21. Plummer M, et al. JAGS: A program for analysis of Bayesian graphical models using Gibbs sampling. In Proceedings of the 3rd international workshop on distributed statistical computing. vol. 124. Vienna, Austria; 2003. p. 125.

22. Kaminskiy MP, Krivtsov VV. A simple procedure for Bayesian estimation of the Weibull distribution. IEEE Transactions on Reliability. 2005;54(4):612-616.

23. Bogaerts K, Komarek A, Lesaffre E. Survival analysis with interval-censored data: A practical approach with examples in R, SAS, and BUGS. Boca Raton, FL: CRC Press; 2017.

24. Andersen PK, Abildstrom SZ, Rosth $\varnothing \mathrm{j}$ S. Competing risks as a multi-state model. Statistical methods in medical research. 2002;11(2):203-215.

25. Andersen PK, Keiding N. Multi-state models for event history analysis. Statistical methods in medical research. 2002;11(2):91-115.

26. Davison AC, Hinkley DV. Bootstrap Methods and their Application. Cambridge Series in Statistical and Probabilistic Mathematics. Cambridge, UK: Cambridge University Press; 1997.

27. Office for National Statistics. Deaths registered weekly in England and Wales, provisional: week ending 3 April 2020, Release date 14 April 2020. [Webpage]; 2020. Available from:

https://www.ons.gov.uk/peoplepopulationandcommunity/birthsdeathsandmarriages/deaths/ bulletins/deathsregisteredweeklyinenglandandwalesprovisional/weekending3april2020. Accessed $01 / 06 / 2020$

28. Putter H. Tutorial in biostatistics: Competing risks and multi-state models Analyses using the mstate package. Companion file for the mstate package. 2011;.

\section{Figures}

Figure 1 A schematic representation of the possible hospital pathways considered by our methods; at any given time, patients are considered to be in one of the five following states: Acute Ward, Critical Care, Stepdown Ward, Discharge or Mortality.

Figure 2 Overall Length of Stay mean estimates with 50\% and 95\% Predictive Intervals (PI). For CHESS data, the intervals are based on empirical percentiles. Source: own elaboration using CHESS and MFT data.

Figure 3 Output of our simulation for transition parameters estimated using each of our three methods, starting from 23 February, which we take to be the start of the outbreak in the UK. Source: own elaboration using CHESS and MFT data. 
Figure 4 Mean Length of Stay by age and week of admission with $50 \%$ and $95 \%$ Predictive Intervals (PI). Source: own elaboration using CHESS data for England.

Figure 5 Percentage of values that are missing or recorded as unknown for the variables of the raw CHESS data that are used in this paper (Date and week of Hospital Admission, Was in ICU?, ICU Admission date - conditional on the patient being admitted to ICU, Final outcome date, Sex, and Age). Source: own elaboration using CHESS data for England.

Figure 6 Output of our simulation using the MS method (black cloud of points) on MFT data (red lines). Source: own elaboration using MFT data.

Appendix A: Data generating process

The data that we analyse from MFT is routinely collected by the hospital administration teams. Details from doctors' notes and patient admissions are entered into the Trust's Patient Administration System (PAS). Patient data from PAS and WardWatcher are then aggregated together to determine an individual's entire hospitalisation pathway. We further make use of data collected by MFT on testing for COVID-19; this is, again, collected and entered manually into a database called Telepath, which is subsequently joined to the main Trust database by the Trust's data warehouse.

In addition to this routinely collected information, trusts have also been required by Public Health England (PHE) to report individual-level data on patients receiving care for acute respiratory infection and aggregate data on all COVID-19 admissions for CHESS. This information is submitted by 09:00 each day with data corresponding to the previous day. This data is compiled manually, requiring additional input from administrative staff to ensure that the data is sent on time and with the correct information. Information sent to PHE by individual trusts is then compiled into a dataset that is disseminated weekly to trusts and reported on weekly to NHS England.

Appendix B: Value-missingness in CHESS data

Figure 5 presents the percentage of missing values in the raw CHESS data reported by NHS trusts grouped by region. London followed by Midlands have the highest percentages of missing values, while South West obtains the smallest one.

In terms of the variables, final outcome date variable has $33.6 \%$ of missing values. These will be split into cases where the missingness is because the final outcome has not yet happened and those where it has happened but has not been captured.

ICU admission date conditioned on whether the patient was admitted to ICU has $5.36 \%$ missing values. Age has $0.29 \%$ missing values, and date and week of hospital admission only have $0.06 \%$.

By contrast, sex and the variable regarding whether a patient was in ICU or not do not have missing values. However, these do have some recorded values of "unknown" which we interpret as missing. Sex has $0.23 \%$ of unknown values, whereas the item regarding being admitted to ICU has $4.41 \%$.

Appendix C: Data processing

We use CHESS data released on 26 May $2020(N=16,138)$. We first filter out 493 duplicated cases. The de-duplication rule set as follows.

$\begin{array}{ll}\text { Rule } & 0 \\ \text { number of records } & 1 \\ \text { IDS are } & \text { singular } \\ \text { Date/time of admission to ICU } & \text { Any } \\ \text { Other variables } & \text { Any values } \\ \text { Action } & \text { leave unchanged }\end{array}$

Rule

number of records

IDS are

Date/time of admission to ICU

Other variables

Action

Rule

number of records

IDS are

Date/time of admission to ICU

Other variables

Action
1

Identical

Identical values for dateadmittedicu

different values for hospitaladmissiondate

Include record with the earliest hospitaladmissiondate, delete the others

2

2

Identical

Identical values for dateadmittedicu

identical values for hospitaladmissiondate

Include record with the earliest sbdate, delete the others 


Rule
number of records
IDS are
Date/time of admission to ICU
Other variables
Action
Rule
number of records
IDS are
Date/time of admission to ICU
Other variables
Action

Rule

number of records

IDS are

Date/time of admission to ICU

Other variables

Action

Rule

number of records

IDS are

Date/time of admission to ICU

Other variables

Action

Rule

number of records

IDS are

Date/time of admission to ICU

Other variables

Action

Rule

number of records

IDS are

Date/time of admission to ICU

Other variables

Action

Rule

number of records

IDS are

Date/time of admission to ICU

Other variables

Action

Rule

number of records

IDS are

Date/time of admission to ICU

Other variables

Action
3

Identical

Different values for dateadmittedicu

ICU periods are non contiguous

Leave all records in the file but record a different obsid for each record

4

Identical

Different values for dateadmittedicu

ICU periods are contiguous

Merge the records to a single record which has the earliest hospitaladmissiondate and dateadmittedicu and the latest dateleavingicu

5

2

Identical

One of them doesn't have dateadmittedicu

Include record with dateadmittedicu, delete the others

6

2

dentical

Neither has dateadmittedicu

different values for hospitaladmissiondate

Include record with the earliest hospitaladmissiondate, delete the others

7

Identical

Neither has dateadmittedicu

identical values for hospitaladmissiondate

Include record with the earliest sbdate, delete the others

8

Different

Identical values for dateadmittedicu, hoursadmittedicu and minutesadmittedicu (and those values are not missing). identical vales for: sex, ageyear, agemonth, hospitaladmissiondate, trustcode, postcode

Use the record with the earliest estimateddateonset or infectionswabdate, delete the others

9

Identical

identical values for dateadmittedicu for $a$ and $b$, identical values for $c$ and $d$ and $a<c$ $a$ and $c$ have identical values for hospitaladmissiondate as do $b$ and $d$ and $a<b$ delete $b$ and $d$ and run the rule set over $a$ and $c$

10

Identical

$>1$

Any not meeting the above conditions

mark for clerical review

The rules are applied in numerical order. In the small number of cases where rule 10 applies then this will always be where two or more of the rules need to be applied in combination.

We only analyse those patients whose records make explicit that the admission to the hospital unit was due to COVID-19. We make this assumption to exclude nosocomial cases, for whom the LoS begins when they were admitted to hospital for non-COVID-19 reasons. It does not make sense to compare these cases with LoS from COVID-19 hospitalisations. Thus, from 15, 645 deduplicated cases, 8, 938 entries were excluded.

Furthermore, we only analysed cases who were admitted to hospital from 16 March 2020 to 17 May 2020 (i.e. from week 12 to 20). Data before week 12 was omitted as this sample size was small and the treatment policy was different from that in more recent data, with patients having very long lengths of stay early in the outbreak. Data after week 20 was omitted as there are often corrections to historical data from the last week or so, so we could not treat the most recent data as reliable. This removed 317 additional cases. 
Finally the omission of cases due to unknown sex, and negative values in age or recorded age of zero, and unknown (effectively missing) information regarding whether a patient was admitted to ICU or not led to a dataset of 6,208 records. The removal of the records that have an age of 0 recorded needs further explanation. The number of records with age 0 was 725 (which compares to 15 age 1's). Many such records had characteristics that one would not attribute to newborns (e.g. obesity, smoking, diabetes, ulcers etc.). We also note that some cases with ages recorded as 0 in early versions of the CHESS dataset had been updated with non-zero ages by 26 May. It seems likely that the data entry system for CHESS has a default setting of "today" for the DOB and therefore in effect the vast majority of Age 0's were in fact cases where the age/DOB was not available when the data were entered. Hence removing these cases seems prudent.

Some LoS have zero length, where patients enter and leave ICU on the same day and only have to the day of arrival and departure recorded not the time. For such cases, we assumed the outcome occurred half a day after admission, since instantaneous durations are unrealistic. Half a day was chosen so that these cases were not biased to either side of their recorded data. Some cases recorded hourly data for some events but not all, causing some LoS to be in $(-1,0)$. For them, we also adjust the outcome date to half a day after admission. All patients with LoS in $(-\infty,-1]$ were discarded. In total 849 cases had their ICU admission date changed to half a day after hospital admission, 41 cases had the ICU discharge date changed to half a day after ICU admission and 199 cases had final outcome date changed to half a day after hospital admission. Therefore, the choice of how to adjust this data has the largest impact on the hospital admission to ICU length of stay, where this constitutes $28 \%$ of cases. For this length of stay, moving the adjustment to different extremes (either adding 0.1 or 0.9 ) changes the length of stay estimates by no more than one tenth of a day. Therefore, this data processing method does not have a substantial impact on the LoS estimates.

Appendix D: Multi-state survival analysis

Here we present the details of the multi-state survival model used in our analysis. Suppose an individual is in state $u$ at time $t$, then the move that an individual makes to their next state $v$ is governed by a set of transition intensities $q_{u v}(t)=\lim _{\delta t \rightarrow 0} \operatorname{Pr}(S(t+\delta t)=v \mid S(t)=u) / \delta t$. The intensity represents the the instantaneous rate of transition from state $u$ to state $v$.

Data structure and transition-specific parametric models

Given the granularity of routinely collected data in hospitals, all transition times between states are observed exactly, with no additional transitions between observation times. Such data allows us to efficiently model the transition intensities parametrically, which we show here with the use of a Weibull accelerated failure time (AFT) model. It is important to note that the data must first be structured in a specific way. In contrast to standard survival analysis, in the multi-state case, we now have a series of event times $t_{1}, \ldots, t_{n}$ for each individual, corresponding to each change in state. The last of these, may be observed so that the patient has entered an absorbing state i.e. they are discharged or dead, or right censored if the patient is still in the hospital. When there are $n_{u}$ competing events for state $u$, a patient entering state $u$ at time $t_{j}$, has their next event at $t_{j+1}$ which is defined as the minimum time amongst the survival times of the competing events $v_{1}, \ldots, v_{n_{u}}$. A row is created for each transition that is possible for the patient, with an additional column consisting of an indicator corresponding to whether or not the transition is observed or censored at $t_{j+1}$. In this format, we can separate the data by transition and fit a transition-specific parametric model to each subset [19]. Our required data format is described in detail in [28].

Weibull AFT model

In the survival framework, for a random variable $T$, denoting the time until an event of interest occurs, the survival function is given by $S(t)=1-F(t)$, where $F(t)$ is the cumulative density function of $T$. The hazard function, $\lambda(t)$, is defined as the instantaneous the instantaneous rate of occurrence of an event and is given by

$$
\lambda(t)=\lim _{\delta t \rightarrow 0} \frac{P(t \leq T<t+\delta t \mid T \geq t)}{\delta t} .
$$

If we assume that $T \sim$ Weibull $(k, \alpha)$, for shape parameter $k$ and scale $\alpha$, then the baseline survival and hazard functions are given by $\bar{S}(t)=\exp \left(-\alpha t^{k}\right)$ and $\bar{\lambda}(t)=\alpha k t^{k-1}$, respectively.

In an AFT model, covariates, $\boldsymbol{x}$, act multiplicatively on time. This in contrast to the proportional hazards model where the covariates act multiplicatively on the hazard. If we let $\phi_{i}=e^{\boldsymbol{\gamma} \cdot \boldsymbol{x}_{\boldsymbol{i}}}$, where $\boldsymbol{\gamma}$ are the regression coefficients, then we get that

$$
\begin{aligned}
& S_{i}(t)=\exp \left(-\alpha t_{i}^{k} \phi_{i}\right) \\
& \lambda_{i}(t)=\phi_{i} \bar{\lambda}\left(\phi_{i} t\right)=\phi_{i} k\left(\phi_{i} t\right)^{k-1}=\phi_{i}^{k} k t^{k-1} .
\end{aligned}
$$

The model is fit using the maximum likelihood estimation (MLE) method. Formulating the likelihood for a survival model requires the consideration of both the contribution of censored and uncensored individuals. For a potentially right-censored observation, let $c_{i}$ be the event indicator for the $i$ th individual with $c_{i}=1$ if an event occurred and $c_{i}=0$ otherwise. Then the likelihood is given by

$$
L\left(\gamma, k ; t_{i}\right)=\prod_{i=1}^{n} \lambda_{i}\left(t_{i}\right)^{c_{i}} S_{i}\left(t_{i}\right) .
$$


Therefore it follows that the log-likelihood for such a model is given by

$$
\begin{aligned}
\mathcal{L} & =\sum_{i=1}^{n}\left[c_{i} \log \left(\lambda_{i}\left(t_{i}\right)\right)+\log \left(S_{i}\left(t_{i}\right)\right)\right] \\
& =\sum_{i}\left[c_{i}\left(\log \left(k t_{i}^{k-1}\right)+k \log \left(\phi_{i}\right)\right)-\left(\phi_{i} t_{i}\right)^{k}\right] \\
& =\sum_{i}\left[c_{i}\left(\log \left(k t_{i}^{k-1}\right)+k \boldsymbol{\gamma}^{T} \boldsymbol{x}_{\boldsymbol{i}}\right)-t_{i}^{k} e^{k \boldsymbol{\gamma}^{\boldsymbol{T}} \boldsymbol{x}_{\boldsymbol{i}}}\right] .
\end{aligned}
$$

Simulation/Bootstrap

In order to predict time to each transition from all states, we use a Monte Carlo simulation approach. This provides greater flexibility than computing length of stay via an integration, allowing us to predict patient pathways during an outbreak in addition to estimating length of stay in each state. As such, it creates a more powerful planning tool for hospital management. The number of simulated individuals, $N$, is based on COVID-19 positive hospital admissions from MFT since 23 February 2020. Individual survival times are simulated using estimates from each fitted transition-specific model, and iterating through the transition matrix until all patients have reached an absorbing state or are censored at a specified maximum follow-up time. The structure of the simulation treats the simulation as a sequence of competing hazards in the following way.

Let $u$ be the patient's starting state, entered at time $t_{u}=s$ and $t_{\max }$ the maximum follow-up time of interest. For each day of interest, repeat the following to simulate paths for every new admission:

1 Let $V$ be the set of states with an allowed transition from $u$ and $Q_{u}=|V|$ be the number of possible transitions from $u$. While $v \in V$, let $\lambda_{u v}(t)$ be the transition intensity for the transition from $u$ to $v$. Note, if $Q_{u}=0$, we are in an absorbing state and stop.

2 For each possible transition, use the fitted parameter estimates of $\lambda_{u v}(t)$ to simulate a survival time, $\bar{t}_{u v}$.

3 Set $\bar{t}=\min \left\{\bar{t}_{u 1}, \ldots, \bar{t}_{u Q_{u}}, t_{\max }\right\}$. If $\bar{t}=t_{\max }$, stop for this individual.

$4 \quad$ Let $u=z$ for $z \in V$ such that $\bar{t}=t_{u z}$ and set $t_{u}=\bar{t}$

\section{Appendix E: Additional results}

In the main results section, we provided estimates for the LoS until any outcome. This was chosen to facilitate comparison between the different methods. In addition to this LoS, the TC method and the MS method can be used to estimate the length of stay until given outcomes such as discharge or death. ${ }^{[9]}$ In this section, we compare estimates for these LoS. Again, pathways are disaggregated by whether the individual went via ICU. Here we choose to omit further predictor variables such as age or week of admission from the TC method to aid comparison.

Table A1 shows the comparison. For the LoS without ICU, the two methods give similar estimates. Using the TC method on the CHESS data predicts that LoS to mortality without ICU is slightly shorter than that to discharge, whereas the MS model on the MFT data predicts vice versa. This might be explained by the small sample size for the MFT data and the different demographic profile of the wider population captured by CHESS. The two methods predict very different LoS on ICU, with ICU to mortality being a week longer in MS (17.4 days) to that predicted by TC (10.2 days). Similarly, the LoS from ICU to stepdown, where individuals are discharged from ICU back to the general ward, is much longer with the MS model. As with the main results, this could most likely be explained by the presence of a much higher proportion of ECMO patients in the MFT data than in the CHESS data. The LoS from stepdown to discharge is similar for the two methods, with 7.9 from TC and 6.2 from MS. We are unable to estimate stepdown to ICU from CHESS due to the small number of such cases present in the data.

Figure 6 shows the output of our simulator on bootstrapped MFT data using the complete multi-state model in Figure 1 . The red line represents true data that is plotted against 200 bootstrap simulations using fitted estimates for the transitions. Day 0 is taken to be 23 February, which we have taken to be the start of the national outbreak for the UK.

[9] A univariate AFT model is not fit for estimating LoS for competing hazards, such as for death and discharge. 
Table A1 Length of stay estimates to given outcome for England using the truncation corrected (TC) method, and for the Manchester Trust using the multi-state (MS) method. Source: own elaboration using CHESS and MFT data.

\begin{tabular}{ll|cc}
\hline Method & Hospital trajectory & Mean & $95 \%$ Confidence Interval \\
\hline TC & Acute Ward to ICU & 2.0 & $(1.9,2.1)$ \\
TC & Acute Ward to Discharge & 9.4 & $(8.9,9.9)$ \\
TC & Acute Ward to Mortality & 8.3 & $(7.8,8.9)$ \\
TC & ICU to Stepdown & 16.6 & $(15.6,17.6)$ \\
TC & ICU to Mortality & 10.2 & $(9.7,10.7)$ \\
TC & Stepdown to ICU & NA & NA \\
TC & Stepdown to Discharge & 7.9 & $(7.5,8.3)$ \\
\hline Multistate & Acute Ward to ICU & 4.0 & $(2.8,5.0)$ \\
Multistate & Acute Ward to Discharge & 8.8 & $(8.4,10.1)$ \\
Multistate & Acute Ward to Mortality & 11.3 & $(8.8,11.0)$ \\
Multistate & ICU to Stepdown & 24.8 & $(19.0,31.8)$ \\
Multistate & ICU to Mortality & 17.4 & $(14.2,24.4)$ \\
Multistate & Stepdown to ICU & 1.2 & $(0.7,2.3)$ \\
Multistate & Stepdown to Discharge & 6.2 & $(4.9,8.1)$ \\
\hline
\end{tabular}




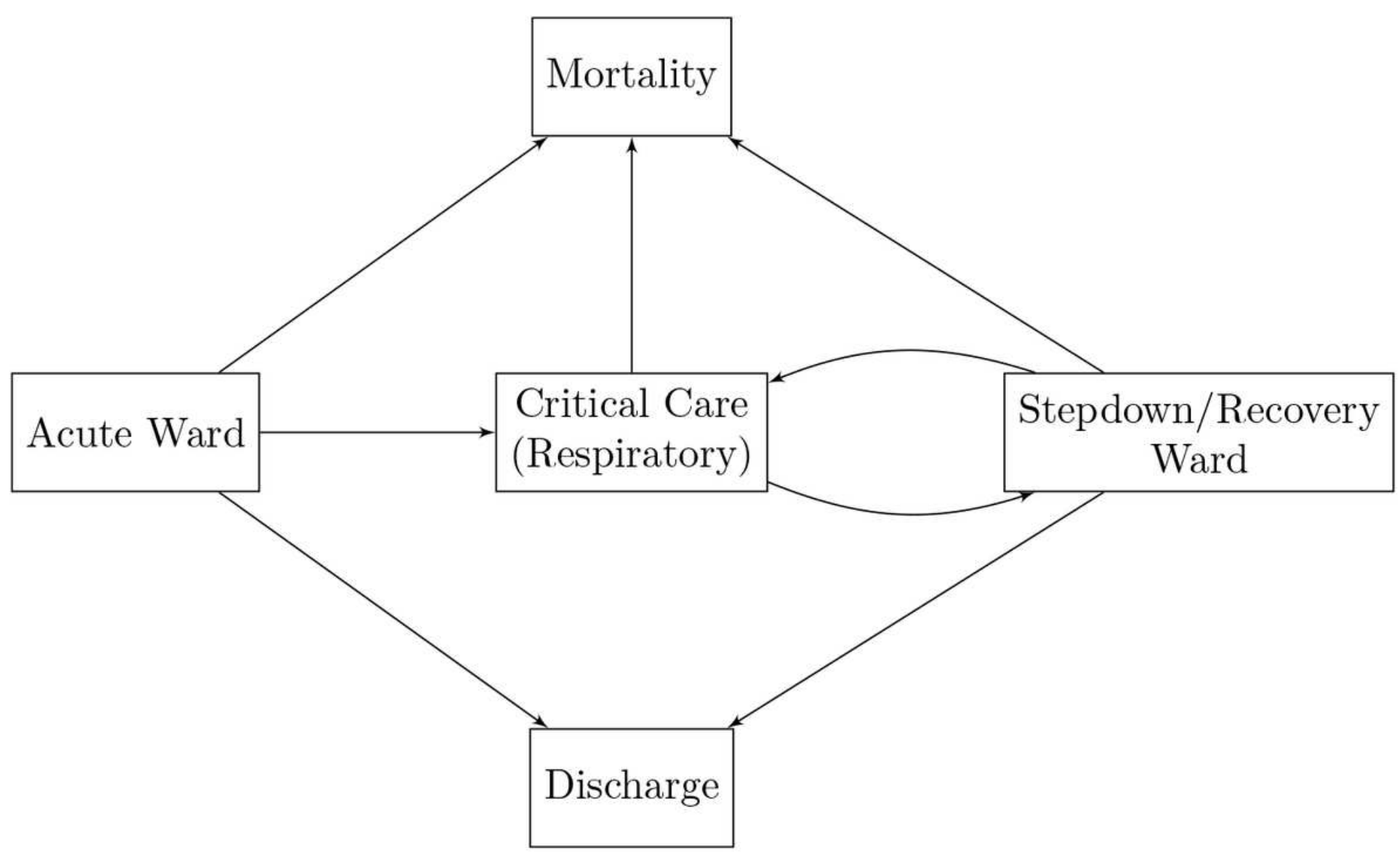

\section{Figure 1}

A schematic representation of the possible hospital pathways considered by our methods; at any given time, patients are considered to be in one of the ve following states: Acute Ward, Critical Care, Stepdown Ward, Discharge or Mortality. 


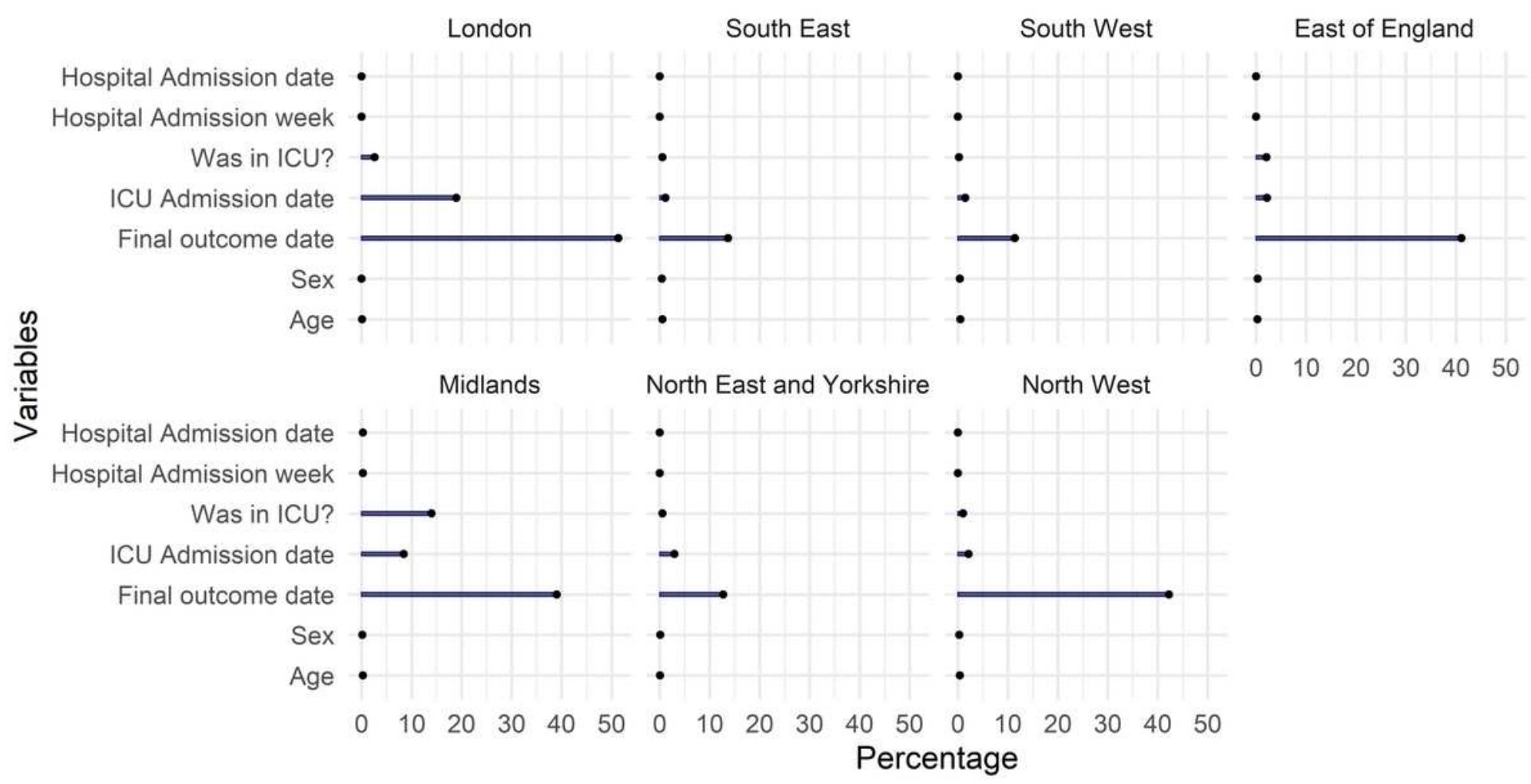

Figure 2

Overall Length of Stay mean estimates with $50 \%$ and $95 \%$ Predictive Intervals (PI). For CHESS data, the intervals are based on empirical percentiles. Source: own elaboration using CHESS and MFT data. 
Multistate Model
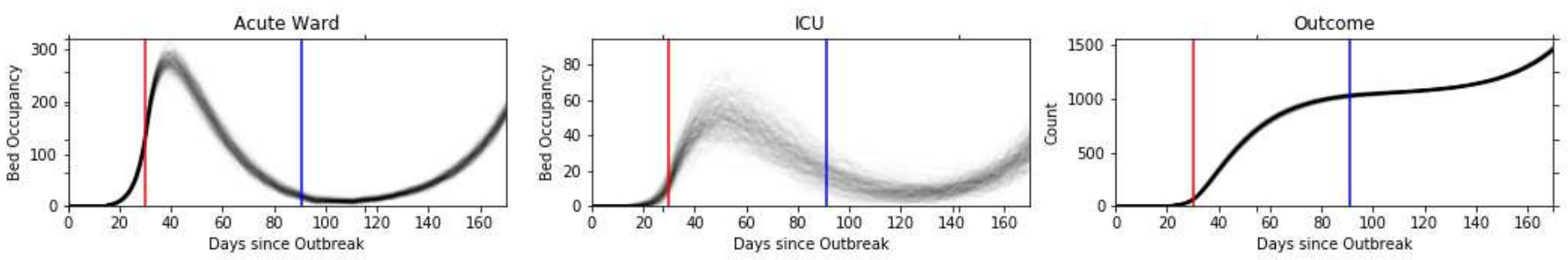

Accelerated Failure Time Model
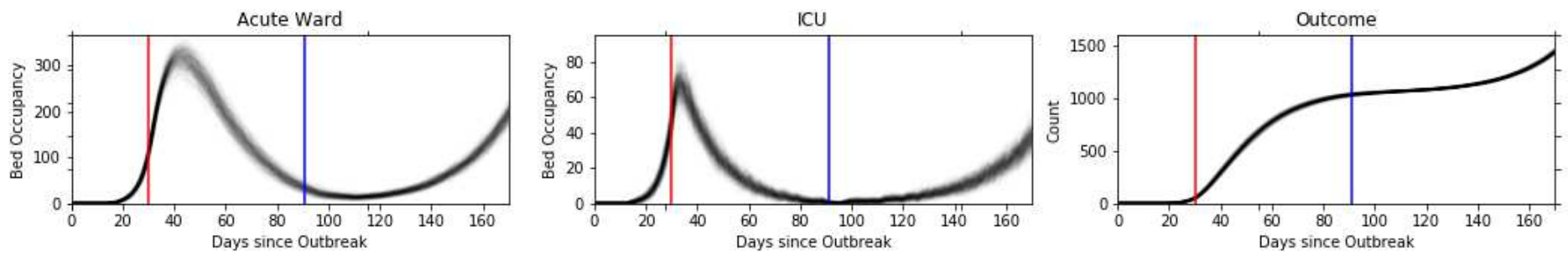

Truncation Corrected Model
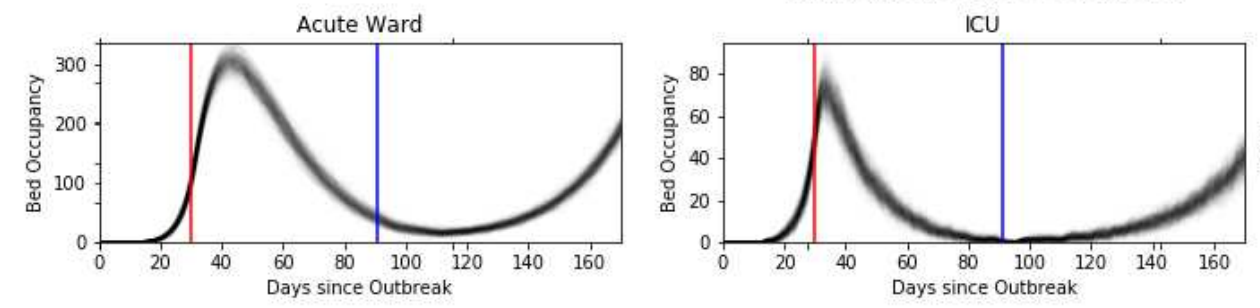

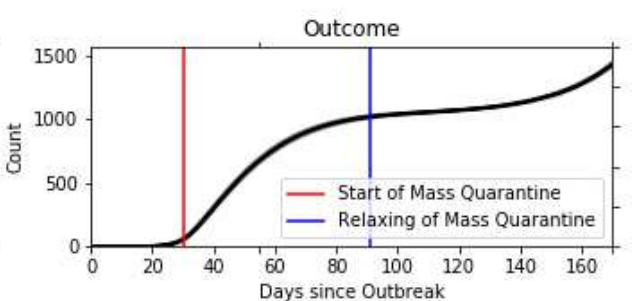

Figure 3

Output of our simulation for transition parameters estimated using each of our three methods, starting from 23 February, which we take to be the start of the outbreak in the UK. Source: own elaboration using CHESS and MFT data. 


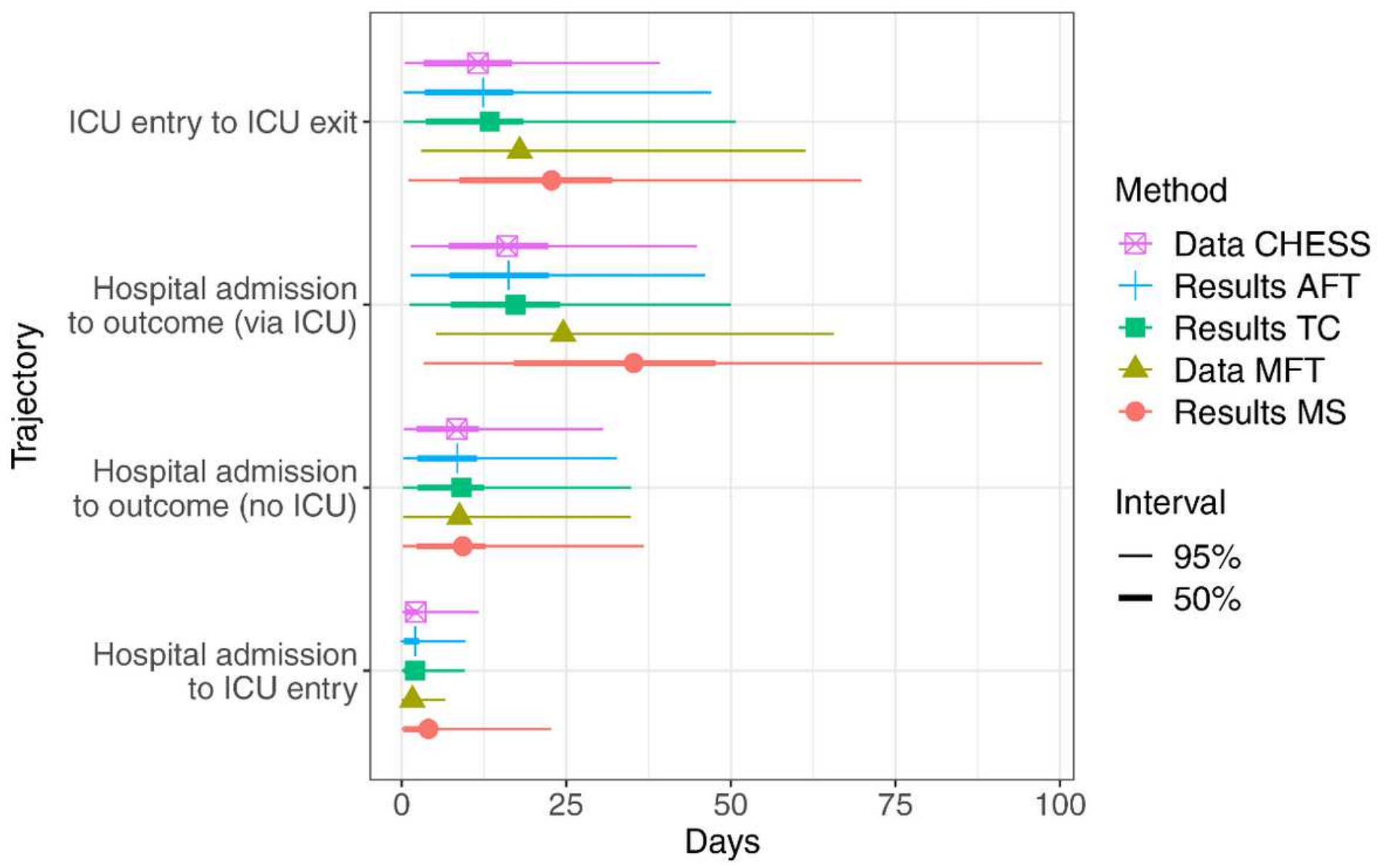

Figure 4

Mean Length of Stay by age and week of admission with $50 \%$ and $95 \%$ Predictive Intervals (PI). Source: own elaboration using CHESS data for England. 


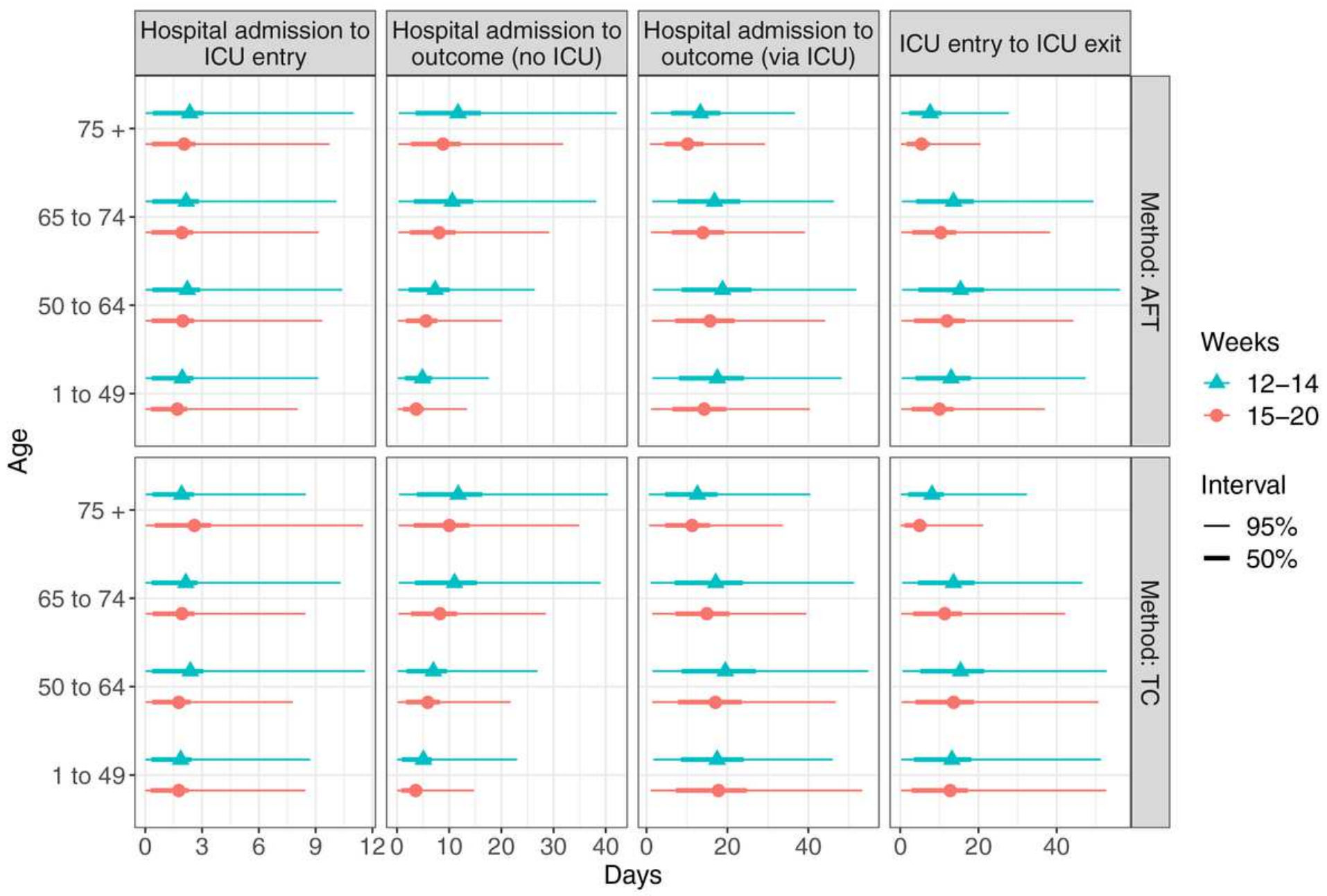

Figure 5

Percentage of values that are missing or recorded as unknown for the variables of the raw CHESS data that are used in this paper (Date and week of Hospital Admission, Was in ICU?, ICU Admission date conditional on the patient being admitted to ICU, Final outcome date, Sex, and Age). Source: own elaboration using CHESS data for England. 

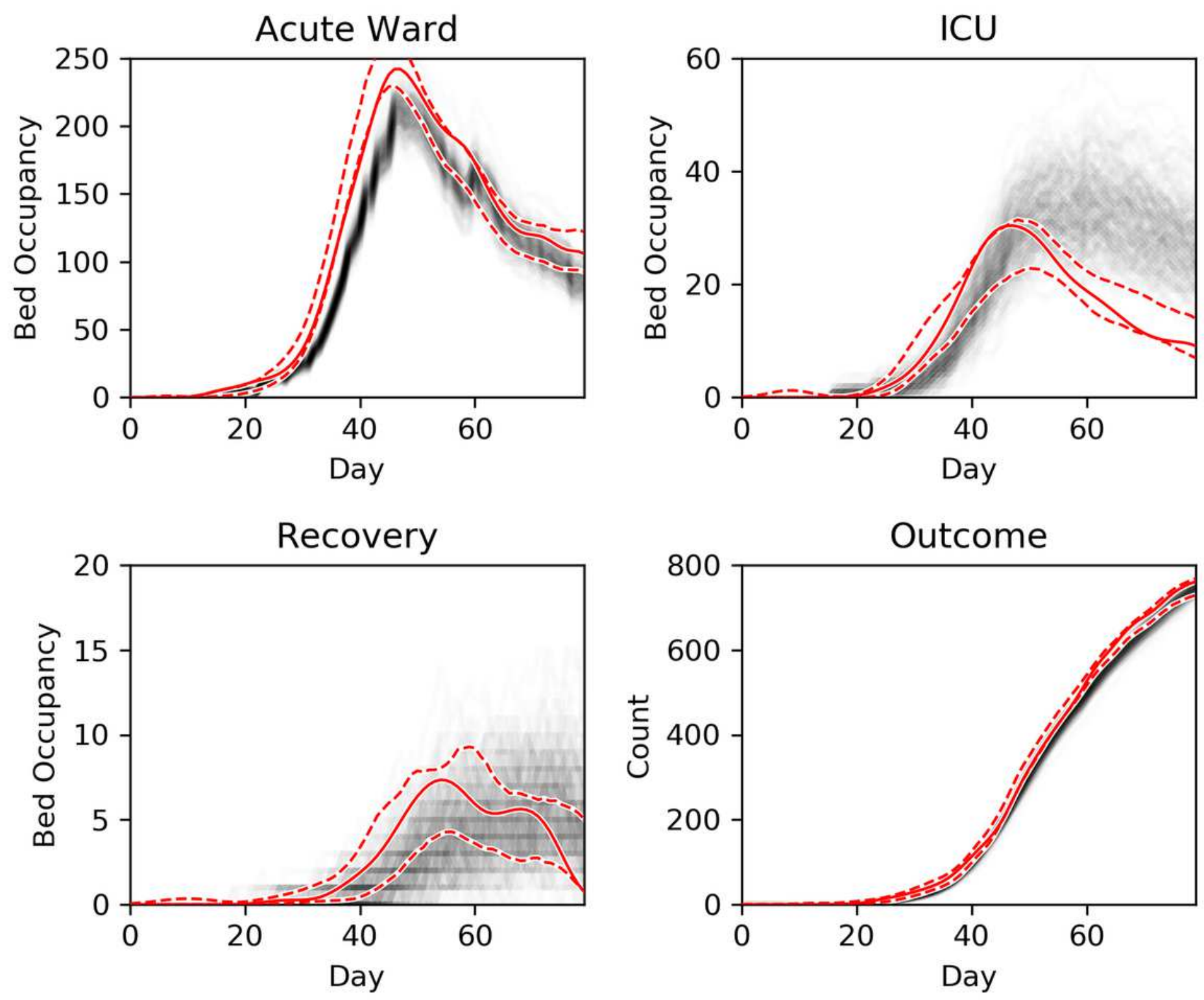

Figure 6

Output of our simulation using the MS method (black cloud of points) on MFT data (red lines). Source: own elaboration using MFT data. 